\title{
DISTRIBUCIÓN GEOGRÁFICA DEL «JEJEO»EN ESPAÑOL Y PROPUESTA DE REFORMULACIÓN Y EXTENSIÓN DEL TÉRMINO
}

\author{
Juan Pablo Rodríguez Prieto \\ Universidad de Florida
}

\section{RESUMEN}

Entendiendo por «jejeo» la sustitución esporádica y condicionada léxicamente de [s] por [h] a principio de sílaba o de palabra, se presenta un resumen de su distribución geográfica en el mundo hispanohablante a través de una revisión de la literatura. Se propone una expansión de su definición incluyendo todas las consonantes fricativas para poder abarcar otros tipos de aspiraciones esporádicas en español que comparten muchas de las características del «jejeo», por ejemplo [hwiste] para «fuiste». También se sugiere que se escriba este término lingüístico con jotas, ya que la práctica de muchos lingüistas es ofrecer ejemplos a medio camino entre las convenciones ortográficas y la transcripción fonética, tales como «nojotros». De esa manera, se establece una mayor correspondencia entre la ortografía y la fonética en esos casos. Finalmente, se mencionarán las causas del estigma social con respecto al uso del «jejeo».

Palabras clave: Jejeo, gegeo, heheo, aspiración, estigmatización, fricativas, inicio de sílaba, inicio de palabra, sustitución esporádica, sustitución léxicamente condicionada.

\section{Abstract}

Understanding Spanish «jejeo» as the lexically conditioned and inconsistent substitution of $[\mathrm{s}]$ for [h] in word and syllable initial position, a summary of its geographical distribution in the Spanish-speaking world is presented through a literature review. An expansion of its definition is proposed by including all fricative sounds in order to account for other sporadic aspirations in Spanish, such as [hwiste] for «fuiste,» that share many characteristics with «jejeo.»It is also suggested that this linguistic feature should be better written using the letter « $\mathrm{j} »$ since many linguists' practice is to provide examples halfway between orthographic conventions and phonetic transcriptions, like «nojotros.» That way, the orthographic-phonetic connection could be better established in those cases. An explanation of the social stigma against the use of «jejeo» is also discussed.

Key Words: Jejeo, gegeo, heheo, aspiration, social linguistic stigmatization, fricatives, sillable initial position, word initial position, sporadic substitution, lexical conditioned substitution. 


\section{INTRODUCCIÓN}

Se entiende por «jejeo» ${ }^{1}$ la sustitución esporádica y condicionada léxicamente de $[\mathrm{s}]$ por [h] a principio de sílaba o de palabra. P. ej.: jeñ ${ }^{2}$ 'señor', $j i$ 'sí'. Es un rasgo lingüístico que no goza de prestigio aunque tiene una distribución amplia en Hispanoamérica, además de encontrarse en el sur de España. Lo suelen emplear hablantes con poco nivel de instrucción o pertenecientes a estratos socioeconómicos bajos, en el habla informal o descuidada. No obstante, también lo utilizan los hablantes cultos en el lenguaje informal/familiar.

El término en sí es muy similar a otros dos procesos de simplificación fonética en español: el «ceceo»y el «seseo». Esta asociación tan simplista podría ser fuente de interpretaciones erróneas del fenómeno del «jejeo». De hecho, Narbona, Cano y Morillo 2003 sugirieron que el nombre de «jejeo» no es el más adecuado para referirse al fenómeno de la aspiración de la /s/ en posición inicial de palabra o de sílaba, ya que da a entender una sistematicidad que casi nunca presenta. Básicamente el «jejeo» solamente afecta a determinadas palabras y muchas veces ni siquiera hay consistencia con una misma palabra. Los autores enfatizaron que se trata de un fenómeno estigmatizado ya que, como ya he mencionado, esta pronunciación está restringida al habla de personas en zonas muy localizadas, de estratos socioculturales bajos y casi siempre en registros descuidados o familiares. Según Narbona, Cano y Morillo 2003, este fenómeno tiene todas las características de un cambio lingüístico embrionario que no ha podido desarrollarse y que, por lo tanto, permanece restringido al habla de determinadas personas y en la producción de ciertas palabras.

El presente trabajo presenta una visión global de la distribución geográfica del «jejeo» en los países de habla hispana a través de una revisión de la literatura. Esta descripción no pretende ser un estudio exhaustivo y minucioso del «jejeo», sino más bien una introducción a las zonas y regiones donde se puede encontrar este rasgo. Por otro lado, dadas las diferencias definitorias con fenómenos lingüísticos análogos tales como el «seseo» o el «ceceo», y dada también la variación terminológica existente para referirse a este fenómeno, se propondrá y justificará una reinterpretación del nombre de «jejeo». Además de ser un intento unificador de la terminología existente («gegeo», «he-

\footnotetext{
${ }^{1}$ Se usa el término «jejeo» escrito con jotas, pese a que se suele encontrar con mayor frecuencia escrito con ges o haches, en base a la propuesta de reformulación y extensión del término que propongo en la sección 3.1. del presente estudio.

${ }^{2}$ Muchos de los ejemplos que se mencionan en este estudio han sido adaptados de los originales, cuyas respectivas fuentes se mencionan, para proporcionar una mayor coherencia y unidad, de nuevo, en base a la propuesta de reformulación del término que expongo más adelante. Es decir, toda sílaba que ejemplifique el fenómeno de «jejeo» se presenta escrita con una jota, siempre y cuando la grafía intente reflejar su pronunciación «jejeísta».
} 
heo», «jejeo» o aspiración de /s / a inicio de palabra o sílaba), es una propuesta que pretende aumentar los contextos lingüísticos en los que puede aplicar la revisada definición del término que, sin duda alguna, va a expandir la distribución geográfica que se presenta a continuación.

\section{Distribución Geográfica del «JEJEO» EN EL MUNDo hispanoha-} BLANTE

\subsection{El «jejeo» en Andalucía (España)}

Lipski 2002 afirma que el español de América tiene muchos rasgos comunes con los principales dialectos de Andalucía. Y, en verdad, si pensamos de manera global en el español de toda Hispanoamérica, vamos a encontrar muchos más rasgos andaluces que castellanos. P. ej.: «seseo» frente a la distinción de $/ \mathrm{s} / \mathrm{y} / \theta /$, realización glotal $[\mathrm{h}]$ de las grafías $j$, gey gi frente a una realización más uvular $[\mathrm{x}], / \mathrm{n} /$ velar a final de palabra [y] en vez de la alveolar [n], etc. Si a este factor añadimos la hegemonía comercial sevillana durante la empresa colonial española de América, es razonable dar validez a las teorías «andalucistas» del español de América que exponen que «el andaluz constituyó el modelo lingüístico más importante durante el periodo de formación del español de América», según aparece en Lipski 2002, p. 50 . El mismo autor también menciona que, una vez que se establecieron ciudades costeras en América y un comercio regular con España, los andaluces tuvieron un papel muy importante en los puertos hispanoamericanos y las zonas costeras adyacentes, pese a que nunca llegaron a ser un número arrollador, a excepción de las regiones costeras. Muchas de estas características comunes pueden ser el resultado de evoluciones posteriores paralelas, pero siempre gracias al influjo andaluz, y no tanto de la exportación andaluza en las primeras décadas del siglo xvi. Por ejemplo, según Vaquero 1996, el «seseo» de signo andaluz acabó imponiéndose en el español de América tras una larga historia de reajustes y valoraciones extralingüísticas, iniciada con la primera nivelación antillana. Canfield 1953 también mencionó que muchas características del español americano, en especial el que se encuentra en las tierras bajas, corresponden a rasgos andaluces actuales y a rasgos andaluces del siglo Xvi. Y es precisamente uno de esos rasgos andaluces de hoy en día, el «jejeo», que también se encuentra en amplias regiones de Hispanoamérica.

En la actualidad, el fenómeno del «jejeo» aparece, según Narbona, Cano y Morillo 2003, de manera esporádica en el habla familiar y relajada de hablantes andaluces de todas las regiones de Andalucía. Pero 
parece que puede encontrarse con cierta sistematicidad en las regiones del sur de Huelva, el centro y sur de Sevilla, Cádiz, la Serranía de Ronda y Málaga capital, en la Subbética cordobesa, en Granada capital y en áreas vecinas (ver mapa 1). Narbona y Morillo 1987 indicaron que formas como jandalia por 'sandalia' están también bastante extendidas en el centro de la provincia de Córdoba. Narbona y Morillo 1987, p. 58 indicaron que esta aspiración prenuclear no está tan extendida como el «seseo» o el «ceceo» y se da en palabras muy localizadas en hablantes aislados y dispersos por el occidente y centro de Andalucía pertenecientes a los estratos socioculturales inferiores.

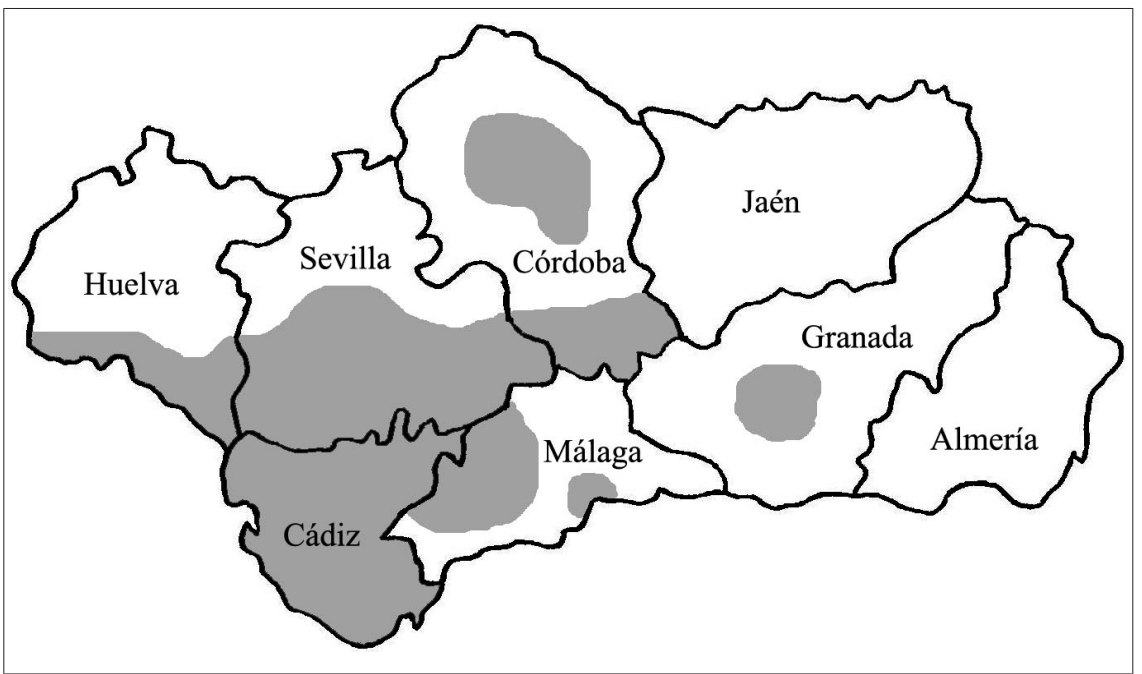

Mapa 1: Distribución geográfica aproximada del «jejeo» en Andalucía, España

\subsection{El «jejeo» en Hispanoamérica}

El «jejeo» es un proceso de relajación articulatoria, y toda relajación articulatoria se debe a la tendencia al menor esfuerzo por parte del hablante. Rosenblat 2002 menciona que en todos los países hispanohablantes de las tierras bajas, es decir, las regiones costeras y las islas, se manifiesta una tendencia al relajamiento en la articulación de la $s$. De hecho, Rosenblat 2002 opina que este rasgo lingüístico es el más importante en la división entre tierras bajas y altas en Hispanoamérica. En muchas regiones de las tierras bajas, la aspiración de $s$ llega a afectar a /s/ intervocálica y a la inicial de sílaba dentro de la cadena hablada, y no solamente a la inicial de palabra. En esta sección se describen las regiones de Hispanoamérica donde podemos encontrar el fenómeno 
del «jejeo», entendido solamente como la sustitución esporádica y léxicamente condicionada de /s/ por [h] a principio de sílaba o de palabra. El análisis está organizado en base a grandes zonas dialectológicas, a saber: el Caribe, Norteamérica, Centroamérica y América del Sur, pero se presentan con más detalle aquellos países donde la incidencia del «jejeo» se ha registrado con mayor frecuencia, pudiéndose considerar incluso como rasgo lingüístico característico de la región. Las principales obras de referencia general del español hablado en Hispanoamérica consultadas son las siguientes: Aleza y Enguita 2002, Alvar 2000, Canfield 1981, Frago y Franco 2003, Lipski 2002, Rosenblat 2002 y Vaquero 1996.

\subsubsection{El «jejeo» en el Caribe}

$\mathrm{El}$ «jejeo» se encuentra en mayor o menor grado en las variedades del español caribeño, pero solamente en los dialectos isleños, es decir, en Cuba, la República Dominicana y Puerto Rico (ver mapa 2). Alvar 1996 y Vaquero 1996 mencionan que, en general, el «jejeo» en Santo Domingo y en el español popular dominicano se da en posición prenuclear intervocálica tanto en interior de palabra como dentro de la cadena hablada: [nõhotro] nosotros, [sĩṇko hẹntaßo] cinco centavos, [la hĕmãnã pahada] la semana pasada, [ehe] ese. Esta aspiración prenuclear también se encuentra, pero de manera esporádica y solamente en estilos de habla muy especiales, en Puerto Rico y Cuba.

\subsubsection{El «jejeo» en Norteamérica}

En el español de América del Norte (mapa 2), podemos encontrar «jejeo» con regularidad en hablantes rurales en el noroeste de México. Los habitantes de las ciudades de esta región mexicana evitan usarlo ya que este rasgo está estigmatizado. Además, su español no difiere mucho del de los hablantes de México central ${ }^{3}$. En cuanto al español hablado en los Estados Unidos, Frago y Franco 2003 al igual que Canfield 1981 identificaron los territorios de Nuevo México, Texas y el sur de Colorado como estados donde el fonema /s/ se aspira en posición prenuclear inicial o interior de palabra: [nohotro] nosotros, [lahabana] la sabana, [lakohina] la cocina. Para Nuevo México, Rosenblat 2002 mencionó los siguientes ejemplos: jiempre, juelta, lo japatoh, la jábanah, etc. Canfield 1981 analizó y fechó el español en esos enclaves como español arcaico de la Andalucía de finales del siglo Xvi.

\footnotetext{
${ }^{3}$ Merece la pena señalar que uno de los evaluadores del presente trabajo también pudo apreciar de manera frecuente ejemplos de este fenómeno en el sur de México.
} 


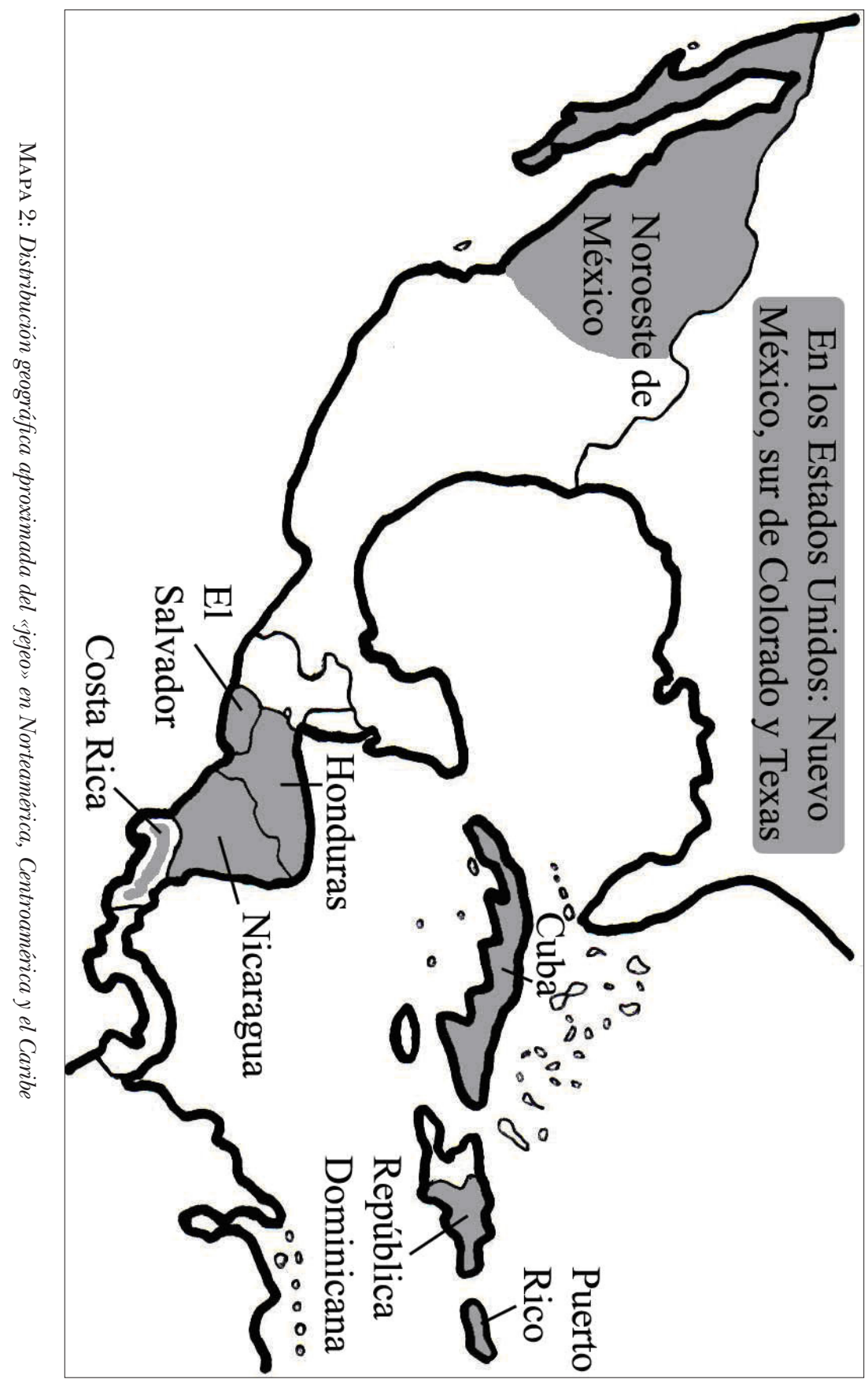




\subsubsection{El «jejeo» en Centroamérica}

Con la excepción de Guatemala, podemos encontrar «jejeo» en toda Centroamérica en mayor o menor grado (ver mapa 2). Frago y Franco 2003 mencionan El Salvador, Costa Rica y Nicaragua como países centroamericanos que aspiran /s/ en posición prenuclear tanto inicial como interior de palabra: [nohotro], nosotros; [lahabana], la sabana; [lakohina], la cocina. El caso de Costa Rica se menciona en Alvar 1996, donde se recoge que el «jejeo»se encuentra en la región central del país en el habla rápida y descuidada, principalmente en casos de disimilación. La disimilación consiste en diferenciar formas iguales o parecidas dentro de un mismo vocablo, en este caso cuando hay dos eses dentro de la misma palabra. Los ejemplos que mencionaron fueron: [nesesarjo] ${ }^{4}>$ [nehesarjo] necesario, [nohotros] nosotros.

Para El Salvador, Lipski 2002 mencionó que el «jejeo»se encuentra entre los estratos socioeconómicos más bajos. Además, el español salvadoreño también reduce a $[\mathrm{h}] \mathrm{la} / \mathrm{s} /$ postconsonántica inicial de palabra en combinaciones como El Jalvador y un jentavo. También destacó la reducción omnipresente de /s / a [h] en el adverbio entonjes. Canfield 1981 cita como ejemplo de «jejeo» salvadoreño el nombre de uno de sus departamentos: [hanta ana] Santa Ana. Geoffroy 1975 propuso la hipótesis de que los ejemplos de «jejeo» salvadoreño son una consecuencia directa del habla de los indígenas nahuat que comenzaron a hablar español una vez establecida la Colonia. Estos indígenas aprendieron a hablar español a través del contacto diario con los conquistadores y no por medio de la enseñanza gramatical en las escuelas. Como consecuencia directa de esta situación de lenguas en contacto, se produjo la deformación de las palabras españolas al suprimir toda diferencia entre $s, c$ y $z$ y sustituirlas por el fonema nahuat velar fricativo sordo que suena como una jota suave: nojotros. Según Geoffroy 1975, este sonido es más notorio y perceptible por los hablantes salvadoreños en el habla de los nicaragüenses. Durante las vacaciones de navidad de 2004/05 y en un viaje que realicé a San Salvador, llevé a cabo una recogida de datos oral en un ambiente distendido a una hablante salvadoreña de la capital de más de 60 años. En un primer análisis de la grabación de voz digital, de unos 50 minutos de duración, pude encontrar claros ejemplos de palabras pronunciadas con «jejeo». A continuación menciono tales ejemplos: $Y$ entonjes ese [jentohehese] día nos dieron el cheque; Ahi abajo, la jeñora [heñora] de ahi abajo tiene un palo de guanaba; Tal vez la otra jemana [hemana] van pa' que vaya a conocer la casa.

Junto con El Salvador, Honduras es el país centroamericano del que más información se tiene sobre el «jejeo», principalmente gracias a los estudios realizados por Lipski 1987, 1990, 2002. En general, las tasas de reducción de /s/ en posición inicial de palabra son menores que

${ }^{4}$ La semivocal palatal va a transcribirse con el símbolo [j] y la semivocal labiovelar con el símbolo $[\mathrm{w}]$. 
las de final de palabra. Aún así se trata de un proceso de cierta envergadura que no se debe al azar. De hecho, Lipski 1987 mencionó que perfectamente se le puede considerar un proceso sistemático en el español hondureño por sus altas tasas de aplicación. Según Lipski 2002, en muchas zonas del país la /s/ inicial de palabra se reduce a [h] en posición intervocálica en el habla continua, p. ej.: la semana [lahemana]. También se debilita la /s/ intervocálica interior de palabra pero en

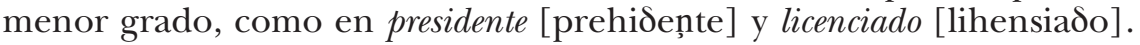
Los ejemplos que Lipski 1987 encontró le hicieron pensar que este tipo de «jejeo» ocurre solamente entre morfemas (pre+supuesto, des+empleo) o en palabras que pareciesen tener una estructura bimorfémica (de-cisión, pre-sidente). También mencionó que la secuencia de dos eses favorece la aspiración inicial de sílaba interior de palabra, por un proceso de disimilación, como ya vimos para el caso de Costa Rica: nejesita, dejisión, lijenciado, etc. Lo que inicialmente pudiese haber surgido en un grupo reducido de vocablos parece haberse extendido a un campo léxico más amplio. Lipski 1987 y 1990 mencionó que los términos que con mayor frecuencia se escuchan con «jejeo» tanto en Honduras como en El Salvador son centavos en combinaciones con otros numerales tales como cincuenta, sesenta, setenta, etc. En su base de datos sobre el habla de Tegucigalpa de 80 entrevistas a hablantes de tres categorías en base a su nivel de escolaridad (poco o nada, nivel de secundaria y nivel universitario), Lipski 1987, p. 113 enumeró las siguientes palabras en las que el «jejeo» era más frecuente: se $(11.7 \%)$, centavo $(10 \%)$, situación $(9 \%)$, cincuenta $(4.7 \%)$, sesenta $(4.6 \%)$, setenta (5\%), San Pedro (Sula) (4.5\%), central $(4.1 \%)$, señor/señora $(3.6 \%)$ y semana $(2.5 \%)$. Según Lipski 2002, parece que, para muchos hablantes estas palabras, se han lexicalizado con una [h] inicial, de tal modo que un centavo puede pronunciarse con $[\mathrm{h}]$ inicial de palabra. La secuencia de fonemas en la que el «jejeo» suele aparecer con mayor frecuencia es $[\mathrm{VhV}]$, independientemente de donde ocurra la división entre palabras, es decir, la posición del límite de palabra no necesita ser especificada con precisión: Vh\#V (/s/ prevocálica final de palabra) o $\mathrm{V \# hV} \mathrm{(/s/} \mathrm{postvocálica} \mathrm{inicial} \mathrm{de} \mathrm{palabra).}$ El «jejeo» hondureño refleja de manera más directa la marginación sociolingüística, pues este rasgo lingüístico predomina entre las clases trabajadoras y los habitantes rurales. Lipski 2002 afirma que es inusual en el nivel más coloquial del habla de los hondureños cultos, o en el habla autoconsciente y cuidada, en la lectura o en el habla en público.

\subsubsection{El «jejeo» en Sudamérica}

Para América del Sur se mencionan Chile, Venezuela y Colombia (ver mapa 3). Frago y Franco 2003 indicaron que el «jejeo» se está ex- 
tendiendo en aquellas regiones en las que apenas se oía, tales como el norte de América del Sur o el interior de algunas regiones centroamericanas, y siempre entre los estratos sociales menos escolarizados. Según Rosenblat 2002, el «jejeo» chileno solamente aparece en los sectores más populares en palabras como meja 'mesa', caja 'casa', coja 'cosa', etc. Por otro lado, en el interior de Venezuela el «jejeo» es ocasional y aparece en formas de desgaste excepcional tales como ji jeñol. Colombia, por su parte, es uno de los países de Hispanoamérica más estudiados y del que se ha publicado un atlas lingüístico gracias al esfuerzo del Instituto Caro y Cuervo. Por esta razón ahora nos concentraremos más en detalle en la distribución geográfica del «jejeo» en Colombia.

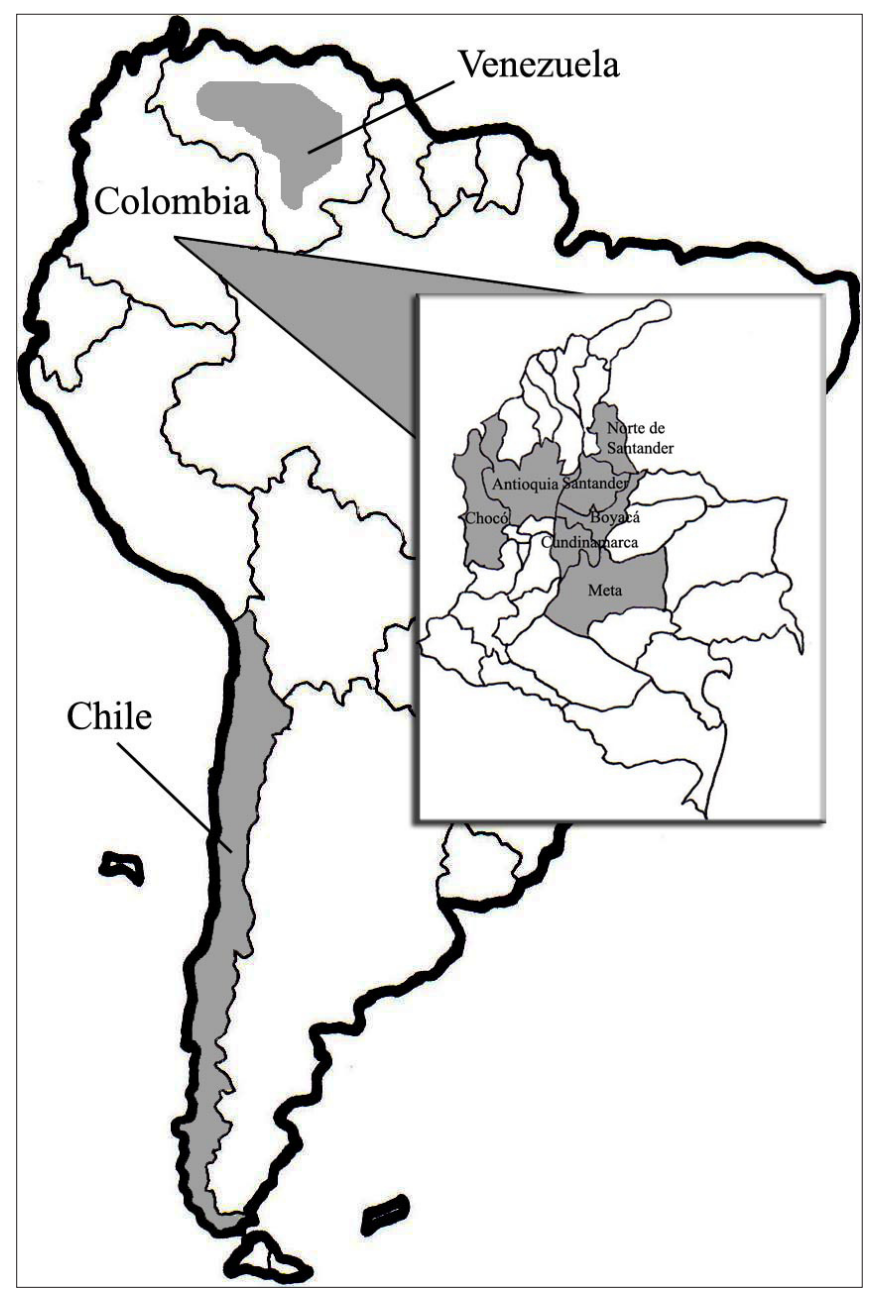

Mapa 3: Distribución geográfica aproximada del «jejeo» en América del Sur 
En las tierras altas del interior de Colombia se conserva /s / final de sílaba y de palabra como [s] sibilante, resistiéndose al debilitamiento, pero Lipski 2002 afirma que a veces /s / se aspira en posición inicial de palabra e intervocálica. La pronunciación de nosotros como nojotros está muy extendida en el centro de Colombia, incluso entre hablantes con un nivel cultural relativamente alto. Esta zona es, por lo tanto, la única del mundo hispanohablante que reduce /s/ más frecuentemente en posición inicial de sílaba que en posición final de sílaba. Esta pronunciación solamente es frecuente en estratos sociolingüísticos muy bajos. Para la costa del Pacífico colombiana, Rosenblat 2002 indica los siguientes ejemplos: no jeñó, sí jeñó, no jalió, no je me quere cayao 'no se me quede callado'; mientras que para las tierras bajas de Antioquia atestigua los siguientes: majamorra, nojotros, munijipal, etc., y también la jal, el jol, la jabaleta, cincuenta jentímetros, etc. Alvar 1996 refiere que la /s/ intervocálica puede elidirse en algunos casos también, como en: [nohotros, nootros]. A continuación nos vamos a concentrar en algunos departamentos en más detalle (ver mapa 3).

Schwegler 1991 ofrece en su trabajo transcripciones del habla vernácula en el departamento del Chocó. Las localidades afro-americanas en las que realizó las grabaciones fueron Viro-Viro, Bagadó y las comunidades Quibdó, Tanguí, Beté y Bella Vista. Schwegler 1991 informa que los choacanos siempre mostraron minusvaloración por su modalidad lingüística local, y en especial en presencia de forasteros. Para todos los hablantes entrevistados observó la realización de /s / final de sílaba y, en algunos casos también, inicial de sílaba, es decir, «jejeo», como [s], [s $\left.\mathrm{h}^{\mathrm{h}}\right]$, $[\mathrm{h}]$ o $[\varnothing]$. A continuación menciono los vocablos que, en algunas oca-

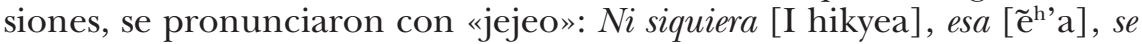
ven $\left[{ }^{\mathrm{h}} \mathrm{e}\right]$, sí sé que $[\mathrm{si} \mathrm{he}]$, dice así $\left[{ }^{\delta} \mathrm{ihe}\right],{ }_{i}$ Es miedoso sí! $\left[{ }^{\mathrm{h}} \mathrm{i}\right]$, sólo $\left[{ }^{\mathrm{h}} \mathrm{olo}\right]$, cosas [koha], setenta [hetẽnta], un peso [peho], nosotros $\left[\right.$ no $^{\mathrm{h}}$ otro] y campesino

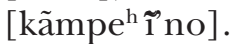

En el departamento de Antioquia, Flórez 1957 descubrió que la $s$ se aspira en posición intervocálica tanto en interior de palabra como en el enlace de unas palabras con otras, aunque con menor frecuencia que en posición implosiva y solamente en el habla rápida y espontánea. Incluso una participante le mencionó que «allá», refiriéndose a Antioquia, jotiaban la $s$. No obstante, parece que esta aspiración es bastante débil y no alcanza a tener tanta fricción como la jota hispanoamericana corriente. Los ejemplos que citó Flórez 1957 en su libro fueron los siguientes: majamorra, nojotros, municipal y máj o menos. Además, la $s$ (o la $c$ ) inicial de sílaba también se aspira, aunque de manera leve y ocasional, en el transcurso de la frase. Los ejemplos ofrecidos fueron los siguientes: la sal: la jal, el sol: el jol, la sabaleta: la jabaleta y cincuenta jentímetros.

En el departamento de Santander, Flórez 1965 afirma que la /s/ tiene ocasionalmente el alófono aspirado [h]. Más específicamente, en 
San Vicente fue capaz de registrar cuatro casos de [h] como pronunciación de /s/ inicial de sílaba en posición intervocálica: un pedazo: peda $\left[\mathrm{s}^{\mathrm{h}}\right] \mathrm{o}$, se hacen: $a ́\left[\mathrm{~s}^{\mathrm{h}}\right]$ en, nojotros y de cemento: de jemento. En general, las personas interrogadas en su estudio fueron de nivel sociocultural bajo, hombres en su mayoría, con edades de entre los 30 a los 75 años. Además, muchos eran campesinos y algunos eran iletrados.

Flórez, Montes y Figueroa 1969 informan que en el departamento del Norte de Santander también se escuchan algunos casos de /s/ intervocálica, tanto interior de palabra como entre palabras, que se relaja y se convierte en una simple aspiración: nojotros (en Cáchira), se me prejentó, la jeñorita (en Pamplona), Dióje lo pague (en Cúcuta).

Por último, Flórez 1951 mencionó que, por lo menos en los departamentos de Cundinamarca, Meta, Boyacá y Santander, hay campesinos que conservan formas antiguas del pretérito del verbo querer: quije, quijo, quijites, quijiera. Para Bogotá, el mismo autor descubrió que en la pronunciación rápida y descuidada se oyen casos de /s/ intervocálica aspirada tales como nojotros, no jeñor, sí jeñor, una jeñora, mi jeñora, yo je lo traje, etc.

\section{Propuesta de Reformulación y eXtensión del término «JejeO»}

Tomando como punto de partida la definición de «jejeo» como aspiración de /s/ en posición inicial de palabra o de sílaba, a continuación expongo las razones por las que considero que este término no se ha sabido utilizar de una manera más precisa y productiva. A su vez, esta sección es un intento de dar coherencia y unidad a un fenómeno lingüístico estigmatizado que ya de por sí es bastante inconsistente y arbitrario. Primero justifico la selección de la convención ortográfica que pudiera dar mayor coherencia a este rasgo lingüístico, para después proponer una extensión de su definición en base a ciertas semejanzas que guarda con otros fenómenos lingüísticos dialectales todavía carentes de una terminología precisa.

\section{1. $\dot{\zeta}$ «egeo», «heheo», «jejeo» o aspiración de /s/ a inicio de palabra o sílaba?}

Comenzando por la ortografía del término en sí, lo primero que llama la atención es la falta de sistematicidad. Podemos encontrarlo escrito con «g», con «h», con «j» o incluso de la manera más pleonástica y descriptiva, es decir, como mera aspiración de /s / a principio de palabra o sílaba. De todas ellas, esta última opción es la que podemos encontrar con mayor frecuencia, aunque también es la menos adecuada, no sólo por cuestiones prácticas sino también porque carece de todo formalis- 
mo. Por otro lado, dado que la letra $h$ del español actual no suele pronunciarse en la mayoría de las variedades del español, creo que el término «heheo» tampoco es una buena opción ya que eso significaría que en realidad se debiera pronunciar [e.eo $]^{5}$. Además, pese a que para el «jejeo» el alófono que representa esta aspiración a principio de palabra se transcribe [h], cuando hablamos de otros fenómenos tales como el «ceceo», se suelen utilizar las convenciones ortográficas y no la fonética para darle su nombre pues, de otro modo, «ceceo» se hubiese escrito

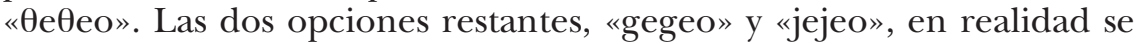
pronuncian de la misma manera pero es la última, en mi opinión, la que ofrece mayor practicidad y consistencia. No cabe duda que muchos lingüistas ofrecen en sus estudios ejemplos de «jejeo» de manera ortográfica y no fonética tales como nohotros, en vez de nosotros con su correspondiente transcripción fonética ([no.hó.troh] donde corresponda). Si comenzásemos a escribir los ejemplos del «jejeo» con jotas y no con haches, tal y como se ha venido realizando en este estudio, estaríamos dando una mayor consistencia ortográfica a este fenómeno de una manera muy sencilla. Al hablar de "gegeo" con ge y ofrecer ejemplos con $h$, creo que es una muestra de inconsistencia. Al mencionar que cuando alguien pronuncia munijipal o jeñor esa persona habla con «jejeo», la consistencia ortográfica salta a la vista. Lo único que habría que tener en cuenta es el menor grado de fricción a la hora de producir dicha jota con pronunciación «jejeísta». Otra razón por la que prefiero «jejeo» frente a «gegeo» es que, si queremos tener mayor consistencia ortográfica, deberíamos escribir los ejemplos con ges del tipo nogotros, magomenos, con lo que perderíamos de inmediato la asociación alofónica de /s/ a [h] por otro alófono inexistente para el «jejeo»: de /s/ a $[\gamma]$.

En resumen, propongo que se utilice el término «jejeo» con jota, ya que la práctica de muchos lingüistas es escribir ejemplos del fenómeno a medio camino entre las convenciones ortográficas y la transcripción fonética. Así p. ej., estos autores escriben nojotros en vez del pronombre nosotros y su transcripción fonética con la aspiración correspondiente al «jejeo», es decir: [no.ho.troh]. Para los hablantes «jejeístas», esta opción ofrece mayor correspondencia entre la ortografía y la articulación de la /s/ como [h] que otras alternativas tales como magamorras o mahamorras.

\section{2. ¿Es el «jejeo» exclusivo para el fonema /s/?}

A continuación propongo una reformulación de la definición del «jejeo» para ampliar los contextos en los que este fenómeno se aplica, partiendo de la definición de otros fenómenos lingüísticos cuyas carac-

${ }^{5}$ Los puntos dentro de la trascripción fonética representan la división silábica. 
terísticas son similares desde una perspectiva puramente formal. Para cada parte de esta nueva definición justificaré las decisiones tomadas. La nueva entrada léxica sería:

Jejeo m. Sustitución esporádica y condicionada léxicamente de las consonantes fricativas por [h] a principio de palabra o de sílaba: [hwis.te] 'fuiste',

[he.ma.na] 'semana', etc.

El «jejeo» es un rasgo lingüístico esporádico ya que, a diferencia de otros fenómenos tales como p. ej. la distinción paraguaya $/ \lambda /$ para $l l$ /y/ para $y$, o la velarización de /n/ a final de palabra en Nicaragua, no se aplica de manera consistente. Una palabra puede pronunciarse con «jejeo» en un momento dado y dos minutos después la misma palabra se articula sin «jejeo» por el mismo hablante. Podemos encontrar ejemplos de esta característica en las transcripciones ofrecidas por Schwegler 1991 en hablantes del Chocó, en Colombia. En ese estudio, el informante de la localidad de Viro-Viro dijo en un momento dado de la grabación: Yo no lo sé [se] no. No lo sé [se], ni el nacimiento... pero unas frases más adelante pronunció la misma forma verbal del verbo saber con «jejeo»: Yo no los vi nunca no; sí sé [he] que bailaron, en base a las transcripciones ofrecidas por Schwegler 1991, pp. 98-99. Tal inconsistencia se puede encontrar incluso en palabras que se pronuncian casi seguidas la una de la otra. En ese mismo estudio, el hablante de Bagadó dijo lo siguiente en un momento dado: Entonces dice [ $\left.{ }^{\complement} \hat{h} \mathrm{he}\right]$ así... donde está el alcalde... dice

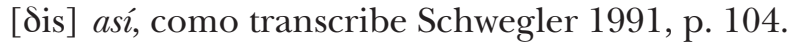

En la revisión de la literatura sobre el «jejeo» presentada al inicio de este estudio se ha hecho notorio que este fenómeno está léxicamente condicionado, es decir, que solamente ocurre en una serie de palabras y que no se aplica en absoluto a toda sílaba o palabra que comienza con el fonema /s/. De hecho, la mayoría de las fuentes consultadas siempre presentan un grupo reducido de ejemplos que, en algunos casos, se trata de los únicos vocablos que encontraron con «jejeo». De entre todas las palabras, es el pronombre personal de sujeto nosotros el que se menciona con mayor frecuencia en todas las variedades estudiadas.

El cambio más importante que propongo respecto a la definición original del «jejeo» es la inclusión de todas las consonantes fricativas y no sólo de /s/. Este cambio viene motivado por la sencilla razón que no solamente /s/ sufre sustitución por [h] a principio de sílaba o palabra de manera esporádica o condicionada léxicamente. De hecho, muchas de las variedades del español que aspiran otras consonantes fricativas a inicio de palabra o de sílaba son, además, variedades «jejeantes». P. ej.: en Nicaragua, Mántica 1997 transcribió ortográficamente la pronunciación de ciertas palabras que comenzaban con $f$ o $h$ por $j$ para el habla de dos informantes nicaragüenses: Jue 'fue', julano 'fulano', juida 'huida', jueron 'fueron', jui 'fui'. En El Salvador, y citando ejemplos en los libros de Sabino Deodanes, Romero 2003 mencionó que la $f$ y la $h$ se aspira- 
ban por influencia de los hábitos lingüísticos nahuats sobre la lengua española: [ku.hrwa.di.ya] cofradia, [a.hwan] afán, [ha.si.ya] hacia, [hu.

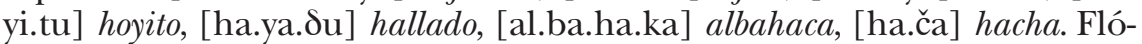
rez 1963 descubrió que en muchos lugares de toda Colombia la gente iletrada solía articular como $j$ la $f$ y la $h$ de la escritura: jamilia 'familia', jogón 'fogón', cajé 'café', jumar 'fumar', mojoso 'mohoso', etc. Como señalaron Flórez, Montes y Figueroa 1969, en el departamento del Norte de Santander, Colombia, encontraron casos más o menos numerosos y frecuentes de $f$ aspirada en el habla inculta: jamilia 'familia', cajé 'café', jiestas 'fiestas', jornicar 'fornicar', dijuntos 'difuntos', jlauta 'flauta', jreno 'freno', etc. Todos estos ejemplos carecen de una terminología precisa que los defina. De hecho, la única diferencia que guardan respecto a los ejemplos de palabras con «jejeo» es la consonante inicial que se aspira. Y no me parece arbitrario que todos estos vocablos comiencen por una consonante fricativa. La definición de «jejeo» propuesta en este estudio abrazaría todos estos ejemplos y a todos aquellos vocablos que se pronuncien con aspiración cuando tengan cualquier consonante fricativa a inicio de palabra o de sílaba.

Quizá pueda llamar la atención que se aspire una $s$ en inicio de sílaba o de palabra, en vez de al final. Pero, en verdad, ni siquiera todas las /s/ finales de palabra o de sílaba se pronuncian en posición posnuclear dentro de la cadena hablada. Cuando se menciona la aspiración de /s/ implosiva se hace referencia a la posición que el fonema ocupa dentro de la palabra o sílaba escrita, no a la silabificación en la cadena hablada. En el sintagma nominal los otros, ambas palabras terminan en $s$ y se considera que la sílaba final de cada palabra tiene el fonema /s/ en posición de coda. Pero eso es cierto solamente si prestamos atención a la ortografía, porque el patrón silábico preferido del español es CV y las sílabas abiertas o que terminan en vocal. Es decir, cuando tenemos una sola consonante en posición intervocálica se suele silabificar con la vocal siguiente. De esta manera, dicho sintagma se pronunciaría [lo.ho.troh] y no [loh.o.troh], a menos que se tratase de una pronunciación enfática. Es decir, el fonema /s/ que en un principio estaba a final de sílaba y palabra en el artículo definido los, en realidad se produce a inicio de sílaba si prestamos atención a la fonética articulatoria y los patrones de silabificación del español, dejando a un lado las convenciones ortográficas. Lo importante del caso es que la pronunciación del pronombre de sujeto nosotros con «jejeo», es decir [no.ho.troh], se percibe como vulgar y estigmatizado. Pero, por otro lado, es aceptable la pronunciación de los otros con aspiración en los dos fonemas /s/ implosivos [lo.ho.troh], percibiéndose como algo natural. En mi opinión, no debería estar estigmatizado el fenómeno del «jejeo», del mismo modo que no se estigmatiza la aspiración de /s / final de sílaba o palabra. De hecho, en algunos ejemplos las diferencias perceptibles en palabras que tienen uno de estos 
dos rasgos lingüísticos son mínimas, como p. ej.: [ma.ha.mor] más amor y [ma.ha.mo.rra] mazamorra. Incluso en algunos casos podría hablarse de pares mínimos en los que solamente uno de los dos miembros estaría estigmatizado, como p. ej.: [no.ho.troh] nosotros y [lo.ho.troh] los otros, siendo la primera una pronunciación estigmatizada y la segunda una pronunciación completamente natural y aceptada. Sin duda alguna, es la falta de sistematicidad con la que aplica este fenómeno lingüístico lo que propicia este valor negativo hacia el «jejeo».

\section{Conclusión}

El «jejeo» es un rasgo lingüístico característico de muchas variedades del mundo hispanohablante pero que está estigmatizado principalmente por el estrato social de la mayoría de los hablantes que lo utilizan, además de ser un fenómeno inconsistente y muy localizado. El incorporar en el estudio del «jejeo» todas las consonantes fricativas del español que sufren un proceso de relajación articulatoria, condicionado léxicamente a inicio de palabra o de sílaba, quizás pudiese ayudar a comprender mejor un rasgo lingüístico tan extendido al igual que tan estigmatizado. Sería muy interesante en futuras investigaciones examinar el habla de diferentes generaciones de hablantes pertenecientes a los estratos socioeconómicos más bajos en algún país «jejeante» y así poder indagar si se trata de un fenómeno en expansión o en declive, tomando como variable independiente cada consonante fricativa de la nueva definición de «jejeo» propuesta en el presente estudio.

\section{REFERENCIAS BIBLIOGRÁFICAS}

Aleza, M. y Enguita, J. M. (2002): El español de América: Aproximación sincrónica, Valencia, Tirant Lo Blanch.

Alvar, M. (1996): Manual de dialectología hispánica. El español de América, Barcelona, Editorial Ariel.

CANFIELD, D. (1953): «Andalucismos en la pronunciación salvadoreña», Hispania 36, pp. 32-33.

- (1981): Spanish pronunciation in the Americas, Chicago, University of Chicago Press.

Flórez, L. (1951): Pronunciación del español en Bogotá, Bogotá, Instituto Caro y Cuervo.

- (1957): Habla y cultura popular en Antioquia, Bogotá, Instituto Caro y Cuervo.

- (1963): «El español hablado en Colombia y su atlas lingüístico», en 1er Congreso de Instituciones Hispánicas, Presente y futuro de la lengua española, Madrid, Ediciones Cultura Hispánica, pp. 5-77.

- (1965): El español hablado en Santander, Bogotá, Instituto Caro y Cuervo. 
- Montes, J. J. y Figueroa, J. (1969): El español hablado en el departamento del Norte de Santander, Bogotá, Instituto Caro y Cuervo.

Frago Gracia, J. A. y Franco Figueroa, M. (2003): El español de América, Cádiz, Servicio de Publicaciones de la Universidad de Cádiz.

Geoffroy Rivas, P. (1975): La lengua salvadoreña. El español que hablamos en El Salvador, San Salvador, Ministerio de Educación.

Lipski, J. (1987): Fonética y fonología del español de Honduras, Tegucigalpa, Editorial Guaymuras.

- (1990): «Reducción de la /s/ en el español de Honduras», en Herranz, A. (ed.), El español hablado en Honduras, Tegucigalpa, Editorial Guaymuras, pp. 91-110.

- (2002): El español de América, Madrid, Cátedra.

Mántica, C. (1997): Introducción al habla nicaragüense, Managua, Editorial Hispamer.

Narbona, A. y Morillo, R. (1987): Las hablas andaluzas, Córdoba, Publicaciones del Monte de Piedad y Caja de Ahorros de Córdoba.

Narbona, A., Cano, R. y Morillo, R. (2003): El español hablado en Andalucía, Sevilla, Fundación José Manuel Lara.

Romero, M. (2003): Diccionario de salvadoreñismos, Santa Tecla, Editorial Delgado. Rosenblat, A. (2002): El español de América, Caracas, Biblioteca Ayacucho.

Schwegler, A. (1991): «El habla cotidiana del Chocó (Colombia)», América Negra 2, pp. 85-119.

VaQuero, M. (1996): El español de América I. Pronunciación, Madrid, Arco/Libros. 